\title{
Utility of 18F-FDG-PET/CT in Lymphoblastic Lymphoma
}

Fox TA ${ }^{1}$, Carpenter B ${ }^{1}$, Taj $\mathrm{M}^{2}$, Perisoglou $\mathrm{M}^{2}$, Nicholson $\mathrm{E}^{2}$, Castleton $\mathrm{A}^{3}$,

Elliot $\mathrm{J}^{3}$, Uttenthal $\mathrm{B}^{4}$, Wright $\mathrm{C}^{4}$, Halsey $\mathrm{R}^{5}$, Khwaja $\mathrm{A}^{1,6}$, Grandage $\mathrm{V}^{1}$, Mansour MR ${ }^{1,6}$, Fielding AK ${ }^{1,6}$, Hough $\mathrm{R}^{1}$

1 - Department of Clinical Haematology, University College London Hospitals NHS Foundation Trust, London, United Kingdom.

2 - Department of Clinical Haematology, Royal Marsden Hospital NHS Foundation Trust, London, United Kingdom

3 - Department of Clinical Haematology, The Christie Hospital NHS Foundation Trust, Manchester, United Kingdom

4 - Department of Clinical Haematology, Cambridge University Hospitals NHS Foundation Trust, Cambridge, United Kingdom

5 - Department of Nuclear Medicine, University College London Hospitals NHS Foundation Trust, London, United Kingdom

6-UCL Cancer Institute, University College London, London, United Kingdom

Corresponding authors:

TA Fox: t.fox@ucl.ac.uk

R Hough: rachaelhough@nhs.net 


\section{Utility of 18F-FDG-PET/CT in Lymphoblastic Lymphoma}

Lymphoblastic lymphoma (LBL) is a rare, malignant disorder of precursor T- or B- cells, which forms the lymphoma variant of acute lymphoblastic leukemia (ALL). Whilst morphologically and immunophenotypically similar to ALL, distinction is made on degree of bone marrow infiltration (less than 25\% in LBL). This makes disease assessment with bone marrow minimal residual disease (MRD) monitoring difficult [1,2].

[18F] Fluoro-2-deoxyglucose (18F-FDG) positron emission tomography CT (PET/CT) is sensitive for staging high grade non-Hodgkin's lymphoma and is superior at differentiating inflammatory from malignant lesions compared to CT alone [3-6]. The utility of 18F-FDGPET/CT in the diagnosis and assessment of LBL is not clear. A prospective study of 76 adult LBL patients found that the results of 18F-FDG-PET/CT after induction therapy did not predict overall survival (OS) [7]. A retrospective Swedish study also concluded that 18F-FDGPET/CT was not predictive of survival although scans were not performed in a uniform manner in this cohort [8]. A small Korean cohort demonstrated the utility of 18F-FDG-PET/CT for evaluation of the extent of disease in LBL but did not assess the predictive value of the modality [9]. A retrospective analysis of data from the GRAAL-LYSA LL03 trial found that higher standardized uptake value $\left(\mathrm{SUV}_{\max }\right)$ at diagnosis was associated with a better prognosis but that $\mathrm{SUV}_{\max }$ after induction was not predictive of OS [10]. There is therefore a strong rationale for further investigation of 18F-FDG-PET/CT in the diagnosis and assessment of LBL.

Patients with a diagnosis of T- or B-cell LBL managed at four tertiary hematology centers in the United Kingdom (University College London Hospital (UCLH), The Royal Marsden 
Hospital, The Christie Hospital and Addenbrooke's Hospital) since 2011 were identified. Data on timing of 18F-FDG-PET/CT scans in patients with a diagnosis of LBL were collected retrospectively and correlated with clinical outcome data. Scans were reported in the tertiary institution at which the patient was treated, and results reviewed in a regional multi-disciplinary team meeting (MDT). Scan and MDT reports were used to establish positive and negative result for this study. A positive study was defined by standard criteria used in lymphoid malignancies, as focal or diffuse uptake above physiological levels in an area incompatible with normal anatomy [11]. Where available, $\mathrm{SUV}_{\max }$ values within the lesion(s) and within the liver, spleen and mediastinal (aortic) blood pool were collected.

44 patients were identified (37 T-LBL, 7 B-LBL) with a median age 24 years (range 10-73 years). 19 patients aged 13-24 years were treated using the UKALL 2011 protocol and 20 aged $>24$ years were treated using the UKALL 14 protocol. Three were treated on the UKALL 2003 protocol and one patient on both the UKALL 12 and UKALL 60+ protocols. 18F-FDG PET/CT was used to varying degrees at different institutions. In the whole cohort, 50\% (22/44) of patients had a 18F-FDG-PET/CT at diagnosis. At several centers, initial investigations were frequently initiated at local hospitals where access to nuclear medicine was limited and 18FDG PET/CT was not performed prior to treatment. Several patients presented in extremis, requiring intensive care support and immediate empirical treatment prior to a $18 \mathrm{~F}-\mathrm{FDG}$ PET/CT being performed. Of the 22 patients who had an 18F-FDG PET/CT at diagnosis, all but one had significant FDG avid disease (mean $\mathrm{SUV}_{\max }$ 8.6, range 2.7-17.1, Tumor-liver ratio >1).

Overall survival (OS) at 2 years for the whole cohort was $86 \%$ (median follow up 33 months). Consistent with previous published data, OS was superior in the younger patients $(96 \%$ in the 
13-24-year age group vs $82 \%$ in those aged $>24$ years) although different patient characteristics and therapy makes these groups incomparable. Of the patients who died, two had a diagnosis of T-LBL and died of disease progression, whilst four of the patients who died had a diagnosis of B-LBL. Of these, two died of disease progression and two due to treatment-related complications (one of neutropenic sepsis/fungal chest infection during consolidation and one due to a myocardial infarction on a background of bacterial pneumonia). Two-year progression free survival (PFS) for the whole cohort was $86 \%$ and $96 \%$ in the T-LBL subgroup ( $n=37)$.

Regardless of age or whether a 18F-FDG-PET/CT was performed at diagnosis, all patients with a negative 18F-FDG-PET/CT scan after cycle one of chemotherapy survived, disease free although it should be noted that the number of patients $(n=5)$ in this group was small (see table 1). Patients with a positive 18F-FDG-PET/CT after course two of treatment or at the end of treatment had a much poorer OS (57\% and $0 \%$ respectively). A complete metabolic remission was obtained at the end of treatment in $91 \%$ of patients. Of the seven patients who had a positive 18F-FDG-PET/CT after the second cycle, five achieved a remission. Three of these seven patients received alloSCT (one of whom died of relapsed disease and two survived). Two continued to respond to treatment and had a negative 18F-FDG-PET/CT at the end of therapy and remain in remission at last follow up. Of those that did not achieve a complete remission, one underwent autologous stem cell transplantation, but both subsequently died of disease progression.

Four patients had primary refractory disease (two of whom had a positive 18F-FDG-PET/CT after course two and two of whom were not imaged after course two). Four patients (9\%) relapsed after achieving a complete metabolic remission, of whom two were successfully re- 
induced and underwent alloSCT whilst two patients relapsed post alloSCT and died of disease progression.

In total six patients underwent allogeneic stem cell transplant (alloSCT) (three patients with a positive 18F-FDG-PET/CT post course two, two with relapsed disease who achieved a second remission and one who was in remission post cycle one who was deemed higher risk due to older age (43)). Four out of these six patients (66\%) were alive post-transplant at last follow up.

In keeping with data from other high-grade lymphomas but contrary to other data in LBL, $S U V_{\max } \geq 10$ at presentation may predict an aggressive clinical course although $S U V_{\max }$ data was only available for 20 patients in this cohort, limiting interpretation (OS 75\% SUV $\max <10$ versus OS 33\% SUV $\max \geq 10, p=0.008$ ) [4,10]. 18F-FDG-PET/CT had 100\% sensitivity and specificity for detecting relapsed disease in symptomatic patients in this small series. In nine patients who presented with symptoms suggestive of disease progression or relapse 18F-FDGPET/CT detected all six patients with true positive disease (confirmed on biopsy) and excluded relapse in three patients with inflammatory lesions.

Our data demonstrates that LBL is FDG-avid in the majority of cases and that 18F-FDGPET/CT is useful in the diagnosis and response assessment of the disease, even if the clinical scenario does not permit a scan at diagnosis. Our findings suggest that the predictive value of 18F-FDG-PET/CT after the first course of treatment is poor but that the predictive value of 18F-FDG-PET/CT after the second cycle of treatment is superior to an assessment after cycle one. Patients who have persistent disease on 18F-FDG-PET/CT after cycle two may benefit from intensification of therapy and consideration given to consolidating remission with 
alloSCT. The predictive value of a 18F-FDG-PET/CT at the end of therapy is good. 18F-FDGPET/CT appears to have a high sensitivity and specificity to detect relapsed disease in a symptomatic patient.

Although patient numbers are small, these data suggest that a high lesion $\mathrm{SUV}_{\max }$ at diagnosis may correlate with an aggressive subtype although this requires verification in a larger cohort. As few patients had a plain CT at the same time as an 18F-FDG-PET/CT, the superiority of 18F-FDG-PET/CT is yet to be established although evidence from other high-grade lymphomas suggests that this warrants further evaluation [12]. Further investigation of the utility of 18F-FDG-PET/CT in risk stratification in LBL is warranted, ideally in the context of a clinical trial.

\section{Author Contributions}

TAF, MP, EN, JE, CW collected the data. TF performed the analysis and wrote the manuscript. All authors were involved in the review of the manuscript and in the clinical care of the patients.

\section{Disclosure of Interest}

The authors report no conflicts of interest. 


\begin{tabular}{|c|c|c|c|c|c|c|}
\hline $\begin{array}{l}\text { Time } \\
\text { Point }\end{array}$ & $\begin{array}{l}\text { Number } \\
\text { of } \\
\text { patients }\end{array}$ & $\begin{array}{l}\text { PET result } \\
\text { (positive/ } \\
\text { negative for } \\
\text { FDG avid } \\
\text { disease) }\end{array}$ & $\begin{array}{l}\text { Number } \\
\text { of } \\
\text { patients }\end{array}$ & $\begin{array}{l}\text { Alive In } \\
\text { remission }\end{array}$ & $\begin{array}{l}\text { Relapse/ } \\
\text { persistent } \\
\text { disease }\end{array}$ & $\begin{array}{l}2 \text { year Overall } \\
\text { survival }\end{array}$ \\
\hline \multirow{2}{*}{$\begin{array}{l}\text { PET at } \\
\text { diagnosis }\end{array}$} & \multirow[t]{2}{*}{22} & Positive & 21 & 16 & 5 & $76 \%$ \\
\hline & & Negative & 1 & 1 & 0 & $100 \%$ \\
\hline \multirow{2}{*}{$\begin{array}{l}\text { PET post } \\
\text { course } 1\end{array}$} & \multirow[t]{2}{*}{19} & Positive & 14 & 10 & 4 & $71 \%$ \\
\hline & & Negative & 5 & 5 & 0 & $100 \%$ \\
\hline \multirow{2}{*}{$\begin{array}{l}\text { PET post } \\
\text { course } 2\end{array}$} & \multirow[t]{2}{*}{20} & Positive & 7 & 4 & 3 & $57 \%$ \\
\hline & & Negative & 13 & 12 & 1 & $92 \%$ \\
\hline \multirow{2}{*}{$\begin{array}{l}\text { PET at } \\
\text { end of } \\
\text { treatment }\end{array}$} & \multirow[t]{2}{*}{23} & Positive & 4 & 0 & 4 & $0 \%$ \\
\hline & & Negative & 19 & 17 & 2 & $89 \%$ \\
\hline \multirow{2}{*}{$\begin{array}{l}\text { PET } \\
\text { performed } \\
\text { to } \\
\text { investigate } \\
\text { symptoms } \\
\text { of possible } \\
\text { relapse }\end{array}$} & \multirow[t]{2}{*}{9} & Positive & 6 & 0 & 6 & $0 \%$ \\
\hline & & Negative & 3 & 3 & 0 & $100 \%$ \\
\hline
\end{tabular}

Table 1: Kaplan-Meier survival analysis in cohorts according to 18F-FDG-PET/CT positivity at different timepoints during treatment. 


\section{References}

1. Cortelazzo S, Ferreri A, Hoelzer D, et al. Lymphoblastic lymphoma. Crit Rev Oncol Hematol. 2017 May;113:304-317.

2. Campana D. Minimal residual disease in acute lymphoblastic leukemia. Semin Hematol. 2009 Jan;46(1):100-6.

3. Ngeow JY, Quek RH, Ng DC, et al. High SUV uptake on FDG-PET/CT predicts for an aggressive B-cell lymphoma in a prospective study of primary FDG-PET/CT staging in lymphoma. Ann Oncol. 2009 Sep;20(9):1543-7.

4. Karantanis D, Durski JM, Lowe VJ, et al. 18F-FDG PET and PET/CT in Burkitt's lymphoma. Eur J Radiol. $2010 \mathrm{Jul} ; 75(1):$ e68-73.

5. Tsukamoto N, Kojima M, Hasegawa M, et al. The usefulness of (18)Ffluorodeoxyglucose positron emission tomography ((18)F-FDG-PET) and a comparison of (18)F-FDG-pet with (67)gallium scintigraphy in the evaluation of lymphoma: relation to histologic subtypes based on the World Health Organization classification. Cancer. 2007 Aug 1;110(3):652-9.

6. Elstrom R, Guan L, Baker G, et al. Utility of FDG-PET scanning in lymphoma by WHO classification. Blood. 2003 May 15;101(10):3875-6.

7. Lepretre $S$, Touzart A, Vermeulin $T$, et al. Pediatric-Like Acute Lymphoblastic Leukemia Therapy in Adults With Lymphoblastic Lymphoma: The GRAALL-LYSA LL03 Study. J Clin Oncol. 2016 Feb 20;34(6):572-80.

8. Ellin $\mathrm{F}$, Jerkeman $\mathrm{M}$, Hagberg $\mathrm{H}$, et al. Treatment outcome in T-cell lymphoblastic lymphoma in adults - a population-based study from the Swedish Lymphoma Registry. Acta Oncol. 2014 Jul;53(7):927-34.

9. Park JH, Pahk K, Kim S, et al. Fluorine-18 fluorodeoxyglucose positron emission tomography imaging of T-lymphoblastic lymphoma patients. Oncol Lett. 2016 Aug;12(2):1620-1622.

10. Becker S, Vermeulin T, Cottereau AS, et al. Predictive value of (18)F-FDG PET/CT in adults with T-cell lymphoblastic lymphoma: post hoc analysis of results from the GRAALL-LYSA LLO3 trial. Eur J Nucl Med Mol Imaging. 2017 Nov;44(12):2034-2041.

11. Kasamon YL, Wahl RL. FDG PET and risk-adapted therapy in Hodgkin's and nonHodgkin's lymphoma. Curr Opin Oncol. 2008 Mar;20(2):206-19.

12. la Fougere $\mathrm{C}$, Hundt W, Brockel N, et al. Value of PET/CT versus PET and CT performed as separate investigations in patients with Hodgkin's disease and nonHodgkin's lymphoma. Eur J Nucl Med Mol Imaging. 2006 Dec;33(12):1417-25. 